\title{
Self Location Estimation Scheme Using ROA in Wireless Sensor Networks
}

\author{
Yun Kyung Lee, Eui Hyeok Kwon, and Jae Sung Lim \\ Graduate School of Information \& Communications, Ajou University, \\ Suwon, Geonggido 443-749, Republic of Korea \\ \{blue9337, k31001, jaslim\} @ajou.ac.kr
}

\begin{abstract}
In wireless channel environments, location estimation error is inevitable when we use the ROA(Received signal strength Of Arrival) for location estimation. Because of multi-path fading and shadowing the received signal strength can not be obtained appropriately in wireless channel. Especially in NLOS(Non-Line Of Sight) environments, the location estimation error is increased significantly. Therefore, it is required to consider the wireless channel to reduce the estimation error. In this paper, we propose a new location estimation scheme to work precisely in NLOS environments. Reference nodes more than three are used to broadcast their location information continuously and each sensor node sorts the average received signal strength considering shadowing characteristics. We use the residual weighting algorithm[1] for ROA location estimation to obtain higher accuracy. As a result, we can obtain accurate location estimation only using location estimation scheme without any special device for location awareness.
\end{abstract}

\section{Introduction}

Recent growth of interest in pervasive computing and location aware system provides a strong motivation to develop the techniques for estimating the location of devices in both outdoor and indoor environments. There have been many approaches to solve this problem[2]. TOA(Time Of Arrival), TDOA(Time Of Difference Arrival), and ROA(Received signal strength Of Arrival) are location aware methods which calculate the relative distance between reference nodes and a sensor node.

TOA uses the time of received signals from the reference nodes to calculate distance. This method requires accurate time synchronization among all of sensor nodes and reference nodes. In case of TDOA, synchronized reference nodes receive signals from a sensor node and calculate time differences between times on which each reference node received signals from the sensor node. However, these methods are hard to be applied to wireless sensor networks since the time or time difference accuracy and synchronization are very sensitive to wireless channel especially in picocell environments.

Existing location aware method based on wireless sensor networks are Centroid[3], APIT[4], and DV-Hop/DV-Distance[5]. Centroid assumes that all signals from reference nodes have the same transmission range and the reference nodes are arranged regularly. So the regular arrangement of reference nodes makes radio transmission range overlapped. And each sensor node recognizes overlapped area as the location of 
sensor node. Although this scheme makes location estimate easy it is difficult to estimate an exact location of a sensor node.

In APIT(Approximate Point In Triangle)[4], reference nodes send location information periodically and each sensor node makes triangles based on the received location information of reference nodes. In this process, a sensor node confirms whether its own location is inside of triangles using received signal strength. And the sensor node recognizes overlapped area of triangles as the location of the sensor node. The defect of APIT is that the precision of location estimate is low and needs a lot of reference nodes because APIT uses self developed method to estimate location. In DVHop/DV-Distance[5], reference nodes transmit their location information periodically and calculate average hop distances using received location information from other reference nodes. And they send the information of average hop distances to neighbor sensor nodes. Sensor nodes which receive this information calculate distance between reference nodes and aware their location. In DV-Distance, instead of transmitting average hop distances, they use signal strength between hops to calculate the distances between reference nodes. However, the location aware method used in DVHop/DV-Distance cannot guarantee accurate location aware.

In this paper we use an ROA method to obtain location awareness adopting the residual weighting algorithm[1] to reduce location estimation error. Besides reference nodes repeat broadcasting their location information and a sensor node categorizes average received signal strength(RSS) according to shadowing property to estimate accurate location awareness. In this method, we assume that signal power loss according to fast fading can be overcame through averaging of received signals. To estimate location a sensor node saves the received signal strengths from each reference nodes more than three. And the sensor node sorts the received signal strength from each reference node to the sensor node based on shadowing characteristics. And then the sensor node calculates the average distance between the reference nodes and the sensor node used by received signal strength which less effecting shadow. After doing range measurement the sensor node estimates its own location using the residual weighting algorithm[1].

The organization of this paper is as follows. In section 2, we explain the residual weighting algorithm using ROA for location estimation. Then simulation environments and simulation results are respectively investigated in section 3 and 4 . Some conclusions are presented in section 5.

\section{Residual Weighting Algorithm Using ROA}

\subsection{Range Measurement Scheme Using ROA}

The observation space shown in Fig. 1 is a set of range measurements, and the parameters that need to estimate location are the geographical coordinates of the sensor node. We assume the random variation of RSS is a log normal Gaussian random variable due to shadowing effect. Thus we can describe that RSS in $\mathrm{dB}$ is distributed with $X_{\sigma_{S}}=N\left(0, \sigma^{2}\right)$ of zero mean and $\sigma^{2}$ variance like Fig. 2. In Fig. 2 each point means an averaged signal strength received from one of reference nodes to the sensor node. When a sensor node estimates an average signal strength from one of reference node 
to the sensor node the sensor node uses the averaged signal strength which is less effecting shadow based on log normal Gaussian distribution and discards the remnant of the averaged received signal strength aren't used to estimate average signal strength. So we can define the ratio of averaged received signal strength samples the sensor node uses to estimate average signal strength. If the number of the averaged received signal strength samples the sensor node saves from one of reference nodes is 100 and the number of averaged received signal strength samples the sensor node uses to estimate average signal strength is 60 based on log normal Gaussian distribution the ratio of averaged received signal strength samples the sensor node uses to estimate average signal strength is 0.6 .

In Fig. 1 the reference nodes(R1 R4) are repeatedly broadcasting data involving its own location information to the sensor node(X). After receiving the signals from each reference node the sensor node sorts the received signal strengths based on shadowing characteristics like Fig. 2 and estimates the average received signal strength used by received signal which is less effecting shadow. And then the sensor node calculates average distances $\left(d_{1} \sim d_{4}\right.$ in Fig.1) from each reference node to the sensor node through average received signal strength.

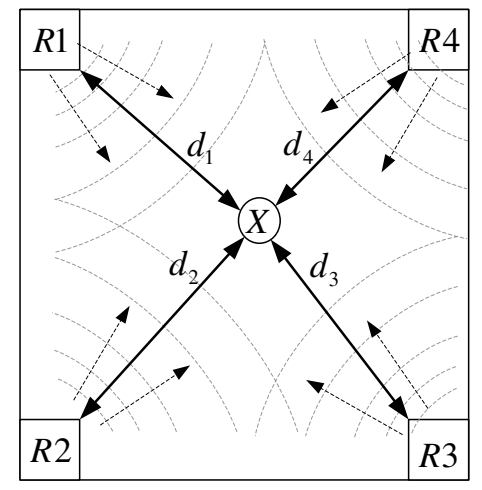

Fig. 1. Referenced Network Topology. Ri : Reference node i, $X$ : Sensor node.

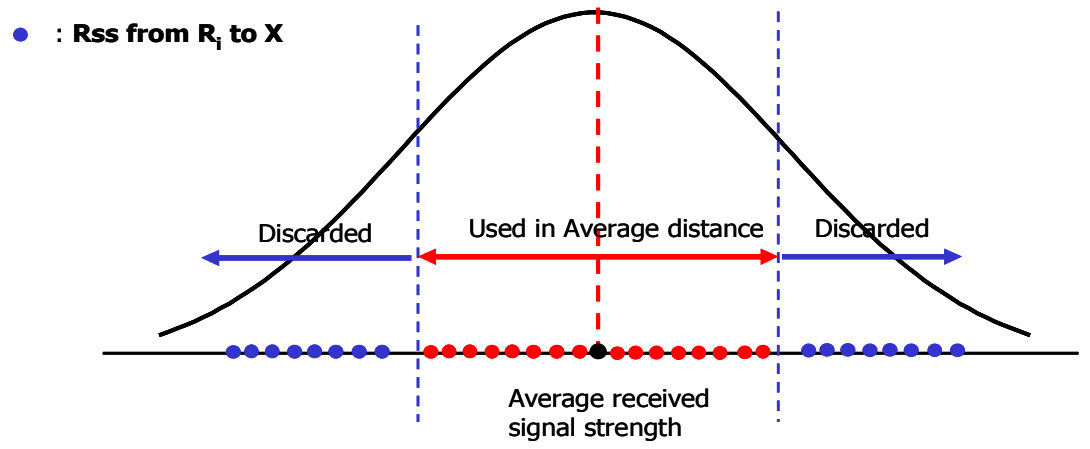

Fig. 2. Distribution of received signal strength 


\subsection{Residual Weighting Algorithm Using ROA}

The residual weighting algorithm[1] was developed to protect location estimate from NLOS error corruption using TOA in the CDMA system. In this paper we use the algorithm to decrease ranging error when a sensor node does a self-location estimation using ROA in shadowing fading channels.

After range measurements using ROA the sensor node of X in Fig. 1 determines the location using LS(Least Square) estimator. That is

$$
\hat{X}=\arg \min \sum_{i \in S}^{N}\left(\left|X-R_{i}\right|-d_{i}\right)^{2}
$$

where

$\left|X-R_{i}\right|$ : distance between vectors $\mathrm{x}$ and $\mathrm{R}_{\mathrm{i}}$

$\mathrm{X}:[\mathrm{x}, \mathrm{y}]^{\mathrm{T}}$, sensor node position in the Cartesian coordinate

$\mathrm{R}_{\mathrm{i}}:\left[\mathrm{X}_{\mathrm{Ri}}, \mathrm{Y}_{\mathrm{Ri}}\right]^{\mathrm{T}}$, reference node position in the Cartesian coordinate

$\mathrm{d}_{\mathrm{i}}$ : the range measurement from the sensor node to the $\mathrm{i}$-th reference node, $i \in s$

$\mathrm{N}$ : the number of reference nodes

$\mathrm{S}:$ the reference node index set

In (1) $\left(\left|X-R_{i}\right|-d_{i}\right)$ is called the $\mathrm{i}$-th residual for the sensor node $\mathrm{X}$. And the range measurement of $\mathrm{d}_{\mathrm{i}}$ is not accurate due to the blocking of the direct path in shadow fading channel.

Equation (1) implies that the LS location estimator, $\hat{X}$, is an estimate which minimizes the sum of the residual squares over the data set, so $\left(\left|X-R_{i}\right|-d_{i}\right)$ can define the residual of the i-th estimated location given by

$$
R_{e s}(\hat{X} ; S)=\sum_{i \in S}\left(\left|\hat{X}-R_{i}\right|-d_{i}\right)^{2} .
$$

Thus, (1) could be rewritten as

$$
\hat{X}=\arg \min R_{e s}(X ; S)
$$

The LS location estimator, $\hat{X}$, is an estimate which minimizes $R_{e S}(X ; S)$. So that is

$$
R_{e S}(\hat{X} ; S)=\min _{X} R_{e S}(X ; S) .
$$

Equation (4) states that a good estimate is the one with minimum residual. When the sensor node does range measurement using ROA, ranging error is always occurred due to shadowing fading channel. So residual $R_{e S}(\hat{X} ; S)$ would not be zero. In the paper we can use a residual $R_{e S}(\hat{X} ; S)$ as the quality indicator of its estimate, $\hat{X}$.

To determine two dimensional location of the sensor node the minimum number of measurements based on ROA is required no less than three. The reference node index set(S) means the group doing range measurement in various ways subject to the constraint with equation (5). For example if the number of reference nodes is $M=4$ (R1 R4) there are 5 eligible range measurement combinations. 
1. Select 4 out of $4:\left(\begin{array}{l}4 \\ 4\end{array}\right)=1$ combination

2. Select 3 out of $4:\left(\begin{array}{l}4 \\ 3\end{array}\right)=4$ combinations

$$
N=\sum_{i=3}^{M}\left(\begin{array}{l}
M \\
i
\end{array}\right)
$$

Therefore there are 5 different range measurement combinations. Applying LS estimator on these combinations using equation (1), we can obtain 5 sensor node estimates, $\hat{X}$, which are denoted as intermediate location estimates.

To use $R_{e s}(\hat{X} ; S)$ as the quality indicator of its estimates, $\hat{X}$, it needs to match the numbers of range measurements in the groups. So the normalized $R_{e s}(\hat{X} ; S)$ is defined as $\widetilde{R}_{e s}(\hat{X} ; S)$ given in (6) to remove the dependence on the size of the group.

$$
\tilde{R}_{e s}(\hat{X} ; S)=\frac{R_{e s}(\hat{X} ; S)}{m}
$$

where $m$ is the size of S. If $\hat{X}$ is obtained by using four reference node in equation (1) the value of $m$ is four and by using three reference node in equation (1) the value of $m$ is three.

Finally, the location of sensor node of $X$ is obtained through equation (7) as the weighted linear combination of the intermediate estimates, $\hat{X}_{1 . . N}$ with its $\widetilde{R}_{e s}\left(\hat{X}_{1 . . N} ; S\right)$. The weight is inversely proportional to $\tilde{R}_{e s}(\hat{X} ; S)$ of the estimate. It means that we can decrease ranging error generated by shadow fading as we could rely more on the estimates derived from those groups which estimates used by signal strengths impacted less effecting shadow.

$$
\hat{X}=\frac{\sum_{k=1}^{N} \hat{X}_{k}\left(\tilde{R}_{e s}\left(\hat{X}_{k} ; S_{k}\right)\right)^{-1}}{\sum_{k=1}^{N}\left(\tilde{R}_{e s}\left(\hat{X}_{k} ; S_{k}\right)\right)^{-1}}
$$

\section{Simulation Environments}

To generate RSS samples as a function of distance the path loss model with the lognormal shadowing effects is used[7]:

$$
P L(d)=30+21 \log _{10}\left(\frac{d}{d_{0}}\right)+X_{\sigma_{S}}
$$


where $P L(d)$ is the path loss for the distance between reference nodes and the sensor node. To consider indoor environment in the simulation we assumed that the value of $\mathrm{d}_{0}$ is $1 \mathrm{~m}$, the path loss exponent $(\eta)$ is 2.1 and path loss for a reference distance is 30 . And the transmit power $\left(\mathrm{P}_{\mathrm{T}}\right)$ of reference nodes is fixed as $10 \mathrm{dBm}$. The random variation of RSS in $\mathrm{dB}$ is expressed as a Gaussian random variable of zero mean and variance of $\sigma^{2}$. In order to generate shadowing effects the value of standard deviation is used $7 \mathrm{~dB}$ in LOS condition and $9.7 \mathrm{~dB}$ in NLOS condition.

To generate NLOS measurements NLOS errors are inserted to range measurements in addition to the measurement errors. In this simulation the exponential distribution with mean $1.5 \mathrm{~dB} \sim 2 \mathrm{~dB}$ as NLOS error model is used. All powers are expressed in $\mathrm{dBm}$ and all distances in meters.

The system model for simulation is Fig. 1. We evaluated the performance of the residual weighting algorithm using ROA in wireless sensor networks when received signals impacted less shadow effects are used. Four reference nodes are broadcasting their location information repeatedly. And a sensor node classifies received signal strengths based on shadowing characteristics and calculates the distances from each reference node to the sensor node using average received signal strength which is obtained by received signal strengths impacted less shadow effects. And the number of samples which a reference node is broadcasting are $20 \sim 100$.

The performance of residual weighting algorithm(Rwgh) using ROA was compared with that of LS estimator(LSE) which is obtained the location of sensor node by only equation (1) using the distances through ROA from each reference node to sensor node. Table 1 is the simulation parameters and ranges for the performance evaluation and ranges.

We used the MATLAB as simulator and the performance criteria of the algorithms as the root mean square error(RMSE) given by

$$
R M S E=\sqrt{\frac{e^{2}{ }_{1}+e^{2} 2 \ldots+e^{2} n}{n-1}}
$$

where $n$ is the number of trials estimating the location of the sensor node and $e_{1 \ldots n}$ is the location error as Euclidean distances between the location estimate and the actual location of the unknown sensor node.

Table 1. Typical values and ranges of simulation parameters

\begin{tabular}{ccc}
\hline Parameters & Typical Value & Typical Range \\
\hline $\mathrm{P}_{\mathrm{T}}$ & $10 \mathrm{dBm}$ & $\mathrm{NA}$ \\
$\mathrm{P}_{\mathrm{L}}\left(\mathrm{d}_{0}\right)$ & $30 \mathrm{~dB}$ & $\mathrm{NA}$ \\
$\eta$ & 2.1 & $\mathrm{NA}$ \\
$\sigma_{S}(\mathrm{LOS})$ & $7 \mathrm{~dB}$ (indoor) & $2-14[7]$ \\
$\sigma_{S}(\mathrm{NLOS})$ & $9.7 \mathrm{~dB}($ indoor $)$ & $2-14[7]$ \\
Dimension & $25 \mathrm{~m} * 25 \mathrm{~m}$ & $\{50,40,30,20\}$ \\
Node & Random & $\mathrm{NA}$ \\
placement & &
\end{tabular}




\section{Simulation Results}

Fig. 3 shows the RMSE according to different dimensions where transmit power is $10 \mathrm{dBm}$, the number of samples which reference nodes is broadcasting is 100 , the values of standard deviation are $7 \mathrm{~dB}$ in LOS condition and $9.7 \mathrm{~dB}$ in NLOS condition for shadowing effects, and the value of mean of NLOS error model is $2 \mathrm{~dB}$. The ratio of averaged received signal strength samples the sensor node uses to estimate average signal strength is 0.6 .

The results show that the value of RMSE is strongly affected by the increase of dimensions due to using ROA to calculate range measurement. In $25 \mathrm{~m} * 25 \mathrm{~m}$ the value of RMSE can be maintained below $2 \mathrm{~m}$ in Rwgh algorithm regardless of LOS and NLOS conditions. It should note that the accurate location estimation can be obtained by ROA in wireless sensor networks.

And in 40m*40m it shows that LOS_Rwgh algorithm is more stable than LOS_LSE because the value of RMSE doesn't exceed 2.5m in LOS_Rwgh algorithm although received signal strength is week against dimension.

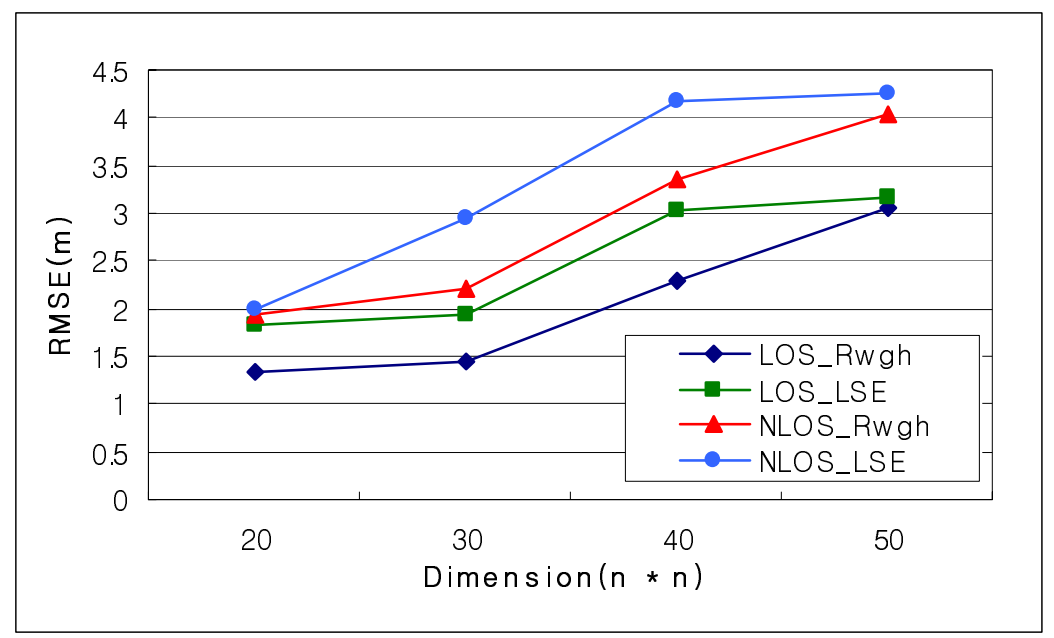

Fig. 3. RMSE according to increase of dimensions

Fig. 4 shows the RMSE for shadowing effects with the different values of standard deviations $\{2,4,6,8,10,12,14\}$ where transmit power of reference node is $10 \mathrm{dBm}$, the dimension is $25 \mathrm{~m} * 25 \mathrm{~m}$, the number of samples which reference nodes is broadcasting is 100, the ratio of averaged received signal strength samples the sensor node uses to estimate average signal strength is 0.6 and the value of mean of NLOS error model is $1.5 \mathrm{~dB}$.

The results show that the value of RMSE doesn't exceed about $2 \mathrm{~m}$ with changes of the value of standard deviation in Rwgh. So it means that we can get accurate location estimation in LOS condition and NLOS condition using ROA. 


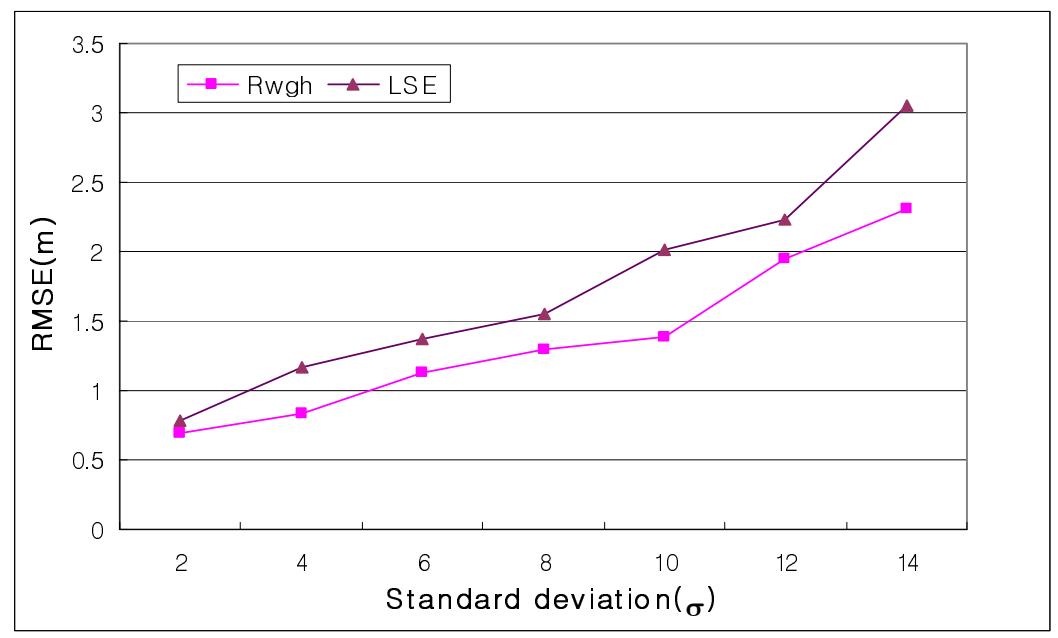

Fig. 4. RMSE according to changes of standard deviations

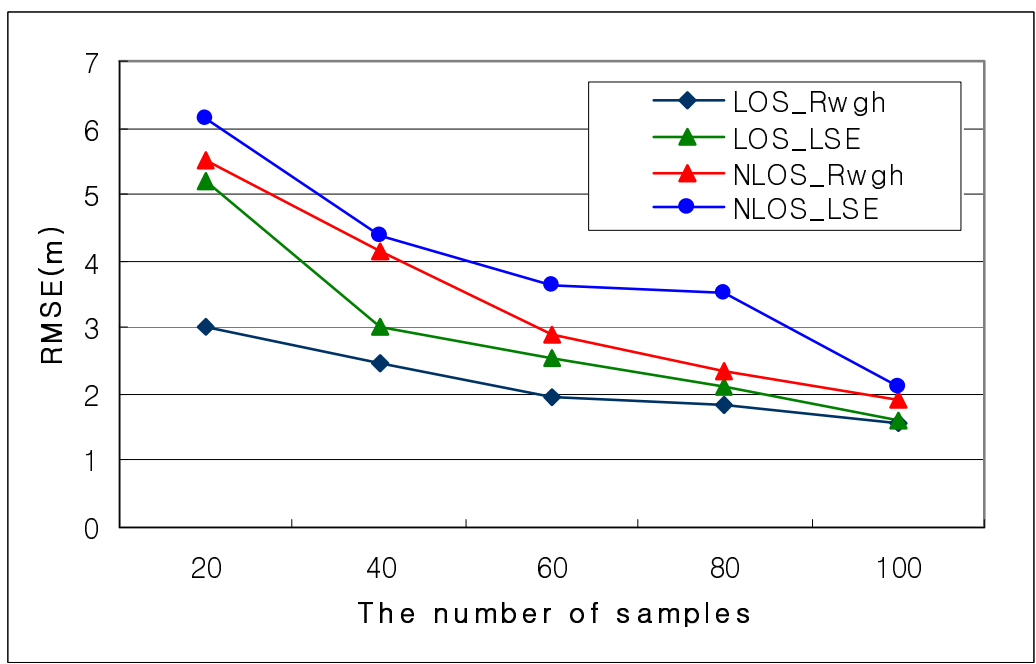

Fig. 5. RMSE for different number of samples

Fig. 5 shows the RMSE for different number of samples. Each RSS sample is generated every packet. In the simulation the dimension is $25 * 25$ and the ratio of averaged received signal strength samples the sensor node uses to estimate average signal strength is 0.8 .

The results show that LOS_Rwgh is more stable than LOS_LSE because the slope of graph in LOS_Rwgh changes slowly. When the number of samples which reference nodes is broadcasting are less than 60 the values of RMSE in NLOS_Rwgh increase almost $4 \mathrm{~m}$. 


\section{Conclusions}

In this paper we proposed a self location estimation scheme using ROA for wireless sensor networks. We can observe through the simulation results that the accurate location estimation can be obtained in LOS/NLOS condition in wireless sensor networks (not exceeding $2.5 \mathrm{~m}$ at dimension of $25 \mathrm{~m} * 25 \mathrm{~m}$ ). And it is possible to get accurate location estimation only using location estimation scheme without any special device for location awareness. But lots of RSS samples are required and the reference nodes should broadcast repeatedly to get more accurate location estimation.

As the future work we are developing a mean value estimation of RSS to obtain high accurate location estimation and an MAC protocol suitable for transmitting location information efficiently.

\section{Acknowledgements}

This work was supported in part by the Ministry of Commerce, Industry and Energy, Korea, grant No.10023210 and in part by grant No. R01200300010724 from the Basic Research Program of the Korea Science \& Engineering Foundation.

\section{References}

1. Pi-Chun Chen: A Non-line of Sight Error Mitigation Algorithm in Location Estimation, IEEE Wireless Communications and Networking Conference, vol. 1, pp. 316-320, 1999.

2. Jochen schiller and Agnes voisard: Location- Based Services, Morgan Kaufmann.

3. N. Bulusu and J. Heidemann: GPS-less Low Cost Outdoor Localization for very small devices, IEEE Personal Communications Magazine, October 2000.

4. Tian He, Chengdu Huang, B. M. Blum, and John A. Stankovic: Range Free Localization Schemes in Large Scale Sensor Networks, Mobicom 2003.

5. D. Niculescu and B. Nath: DV Based Positioning in Ad hoc Networks, Telecommunication Systems, Kluwer, 22(1-4), pp. 267-280, January-April 2003.

6. Asis Nasipuri and Kai Li: A Directionality based Location Discovery Scheme for Wireless Sensor Networks, Wireless Sensor Networks and Applications, September 2002.

7. Rappaport: Wireless Communications principles and practice, Prentice Hall.

8. H. Hashmi: The indoor radio propagation channel. Proceeding of the IEEE, 81(7), pp. 943968, July 1993.

9. F. Mondinelli and Zsolt: Self-Localizing Sensor Network Architectures, IEEE Transactions on Instrumentation and Measurement, vol. 53, no. 2, April 2004.

10. David Christopher Moore: Robust Distributed Sensor Network Localization with Noisy Range Measurements, California Institute of Technology, The ACM conference on Embedded Networked Sensor Systems, November 2004. 